\title{
Synthesis and characterization of core-linked star clusters by conventional free-radical polymerization
}

\author{
Supporting Information
}

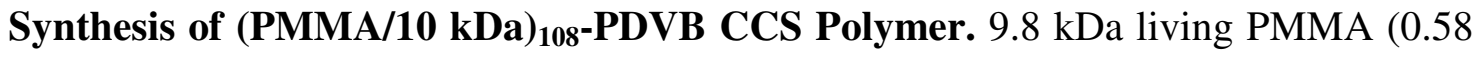
g, $59.0 \mu \mathrm{mol})$, copper(I) chloride $(5.8 \mathrm{mg}, 59.0 \mu \mathrm{mol})$, bypyridine $(28 \mathrm{mg}, 177 \mu \mathrm{mol})$, DVB (113 mg, $0.89 \mathrm{mmol}), 2,2$ '-bipyridine (27.6 mg, $0.18 \mathrm{mmol})$ and $p$-xylene (1.85 $\mathrm{mL}$ ) were added to a Schlenk tube with a magnetic stirrer bar and sealed. The mixture was then subjected to three freeze-pump-thaw cycles and back filled with argon. The tube was heated at $100{ }^{\circ} \mathrm{C}$ for 50 hours. The reaction was quenched with a ten-fold excess of THF and passed through a plug of basic alumina to remove the catalyst followed by precipitation in cold methanol $\left(-18{ }^{\circ} \mathrm{C}\right)$. The precipitate was removed from the mother liquor by vacuum filtration, washed twice with methanol and dried in a dessicator for 5 hours. The CCS polymer mixture was obtained as a white solid, $0.64 \mathrm{~g}(92 \%)$; GPCMALLS, Peak $1(88 \%)-M_{\mathrm{w}}=1,345 \mathrm{kDa}$, PDI $=1.56, f=108, M_{\mathrm{w}, \text { PDVB core }}=291 \mathrm{kDa}$ : Peak $2(12 \%)-M_{\mathrm{w}}=246.1 \mathrm{kDa}, \mathrm{PDI}=1.10$.

Synthesis of (PMMA/21 kDa) 2 -PEGDMA CCS polymer. The synthesis and purification method was consistent to synthesis of (PMMA/10) $)_{108}$-PDVB CCS polymer, however, DVB was substituted for EGDMA (176 mg, $0.89 \mathrm{mmol})$ and $20.7 \mathrm{kDa}$ living PMMA (1.22 g, $59.0 \mu \mathrm{mol})$ was used. The CCS polymer mixture was obtained as a white solid, $0.72 \mathrm{~g}$ (95\%); GPC-MALLS, Peak $1(72 \%)-M_{\mathrm{w}}=530 \mathrm{kDa}$, PDI $=1.06, f$ $=21, M_{\mathrm{w}, \text { PEGDMA core }}=89 \mathrm{kDa}:$ Peak $2(28 \%)-M_{\mathrm{w}}=84.8 \mathrm{kDa}, \mathrm{PDI}=1.72$. 
The number of arms, $f$, and $M_{\mathrm{w}}$ of the core can be calculated as follows. The weight fraction of PMMA arms was calculated by the following equation; $W F_{\text {arms }}=$ mPMA $_{\text {PMA }} /$ $\left(\mathrm{m}_{\text {crosslinker }} \cdot \chi_{\mathrm{C}}+\mathrm{m}_{\text {PMMA }} \cdot \chi_{\mathrm{A}}\right)$, where $\chi_{\mathrm{A}}$ and $\chi_{\mathrm{C}}$ is the conversion of PMMA arms to CCS polymer (determined by GPC analysis) and conversion of crosslinker (determined by GCMS analysis), respectively. The number of arms, $f$, is then calculated as follows; $f=$ $M_{\mathrm{w}, \mathrm{CCS} \text { polymer }} \times W F_{\text {arms }} / M_{\mathrm{w}, \mathrm{PMMA}}$.

Pure CCS polymers were obtained by fractional precipitation, which was carried out by removing excess solvent from the reaction solution in vacuo followed by incremental addition of cold methanol $\left(-18{ }^{\circ} \mathrm{C}\right)$. The precipitate formed was removed from the mother liquor by filtration, washed twice with methanol and dried in a vacuum dessicator $(<0.05$ $\mathrm{mm} \mathrm{Hg}$ ) for 5 hours. The process was repeated with the filtrate until several fractions are obtained. (PMMA/10 kDa) ${ }_{108}$-PDVB CCS Polymer; ${ }^{1} \mathrm{H}$ NMR (400 MHz, $\mathrm{CDCl}_{3}$, TMS) $\delta_{\mathrm{H}}$ 7.75-7.70 ( $\left.m, \operatorname{ArCH}\right), 7.36-7.30(m, \operatorname{ArCH}), 3.73-3.38\left(b r m, \mathrm{OCH}_{3}\right), 2.421(\mathrm{~s}$, $\mathrm{ArCH}_{3}$ ), 2.04-1.68 ( $\left.\mathrm{br} m, \mathrm{CH}_{2}\right), 1.49-1.37$ ( $b r \mathrm{~m}, \mathrm{CH}_{3}$ ), 1.23-1.18 (br m, $\mathrm{CH}_{3}$ ), 0.99 (br s, $\mathrm{CH}_{3}$ ), 0.81 (br s, $\left.\mathrm{CH}_{3}\right)$ ppm; ${ }^{13} \mathrm{C} \mathrm{NMR}\left(100 \mathrm{MHz}, \mathrm{CDCl}_{3}, \mathrm{TMS}\right) \delta_{\mathrm{C}} 178.1(\mathbf{C O}), 177.8$ (CO), $176.9(\mathbf{C O}), 129.9(\mathrm{ArCH}), 127.8(\mathrm{ArCH}), 54.4\left(\mathbf{C H}_{2}\right), 51.8\left(\mathrm{OCH}_{3}\right), 44.9$ $\left(\mathbf{C}\left(\mathrm{CH}_{3}\right)_{2}\right), 44.5\left(\mathbf{C}\left(\mathrm{CH}_{3}\right)_{2}\right), 21.6\left(\mathrm{CH}_{3}\right), 21.0\left(\mathrm{CH}_{3}\right), 18.7\left(\mathbf{C H}_{3}\right), 16.5\left(\mathbf{C H}_{3}\right)$ ppm. (PMMA/21 kDa) 21 -PEGDMA CCS polymer; ${ }^{1} \mathrm{H}$ NMR $\left(400 \mathrm{MHz}, \mathrm{CDCl}_{3}\right) \delta_{\mathrm{H}} 7.73(\mathrm{br}$ $s, \operatorname{ArH}), 7.33(b r s, \operatorname{ArH}), 3.73\left(b r s, \mathrm{OCH}_{3}\right), 3.58$ (br s, $\left.\mathrm{OCH}_{3}\right), 2.43\left(b r s, \mathrm{ArCH}_{3}\right)$, 1.92-1.79 ( $\left.m, \mathrm{CH}_{2}\right), 1.41$ (br s, $\left.\mathbf{C H}_{3}\right), 1.19$ (br s, $\left.\mathbf{C H}_{3}\right), 0.99$ (br s, $\left.\mathbf{C H}_{3}\right), 0.81$ (br s, $\left.\mathrm{CH}_{3}\right)$ ppm; ${ }^{13} \mathrm{C} \mathrm{NMR}\left(100 \mathrm{MHz}, \mathrm{CDCl}_{3}\right) \delta_{\mathrm{C}} 177.8(\mathbf{C O}), 177.5(\mathbf{C O}), 176.6(\mathbf{C O}), 54.2$ 
$\left(\mathrm{OCH}_{3}\right), 54.1\left(\mathrm{OCH}_{3}\right), 54.0\left(\mathrm{OCH}_{3}\right), 51.5\left(\mathrm{CH}_{2}\right), 44.6\left(\mathbf{C}\left(\mathrm{CH}_{3}\right)_{2}\right), 44.2\left(\mathrm{C}_{\left.\left(\mathrm{CH}_{3}\right)_{2}\right), 18.4}\right.$ $\left(\mathrm{CH}_{3}\right), 16.2\left(\mathrm{CH}_{3}\right), 16.1\left(\mathrm{CH}_{3}\right), 16.0\left(\mathrm{CH}_{3}\right) \mathrm{ppm}$.

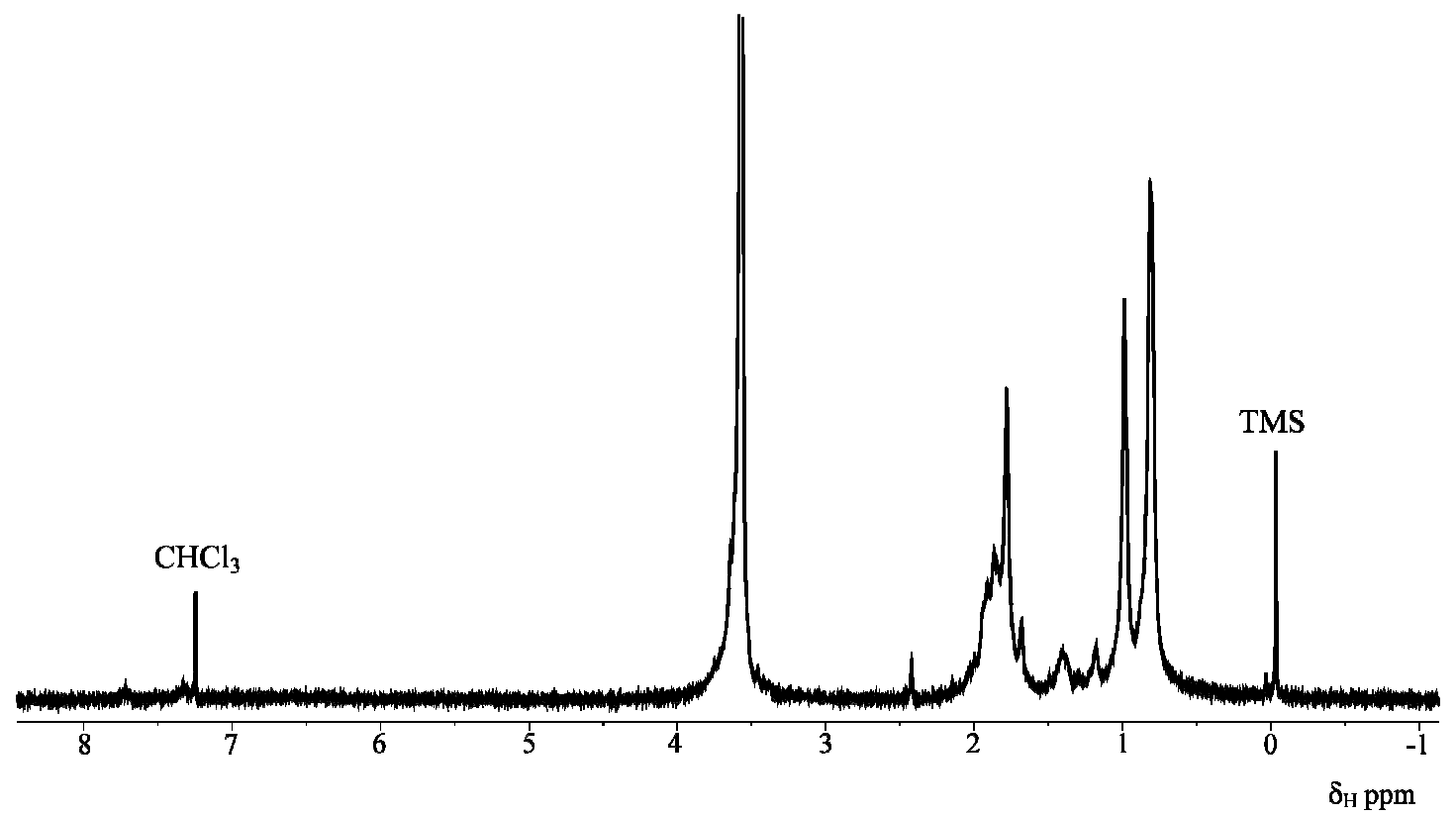

Figure S1. ${ }^{1} \mathrm{H}$ NMR of (PMMA/10 kDa) ${ }_{108}$-PDVB CCS polymer.

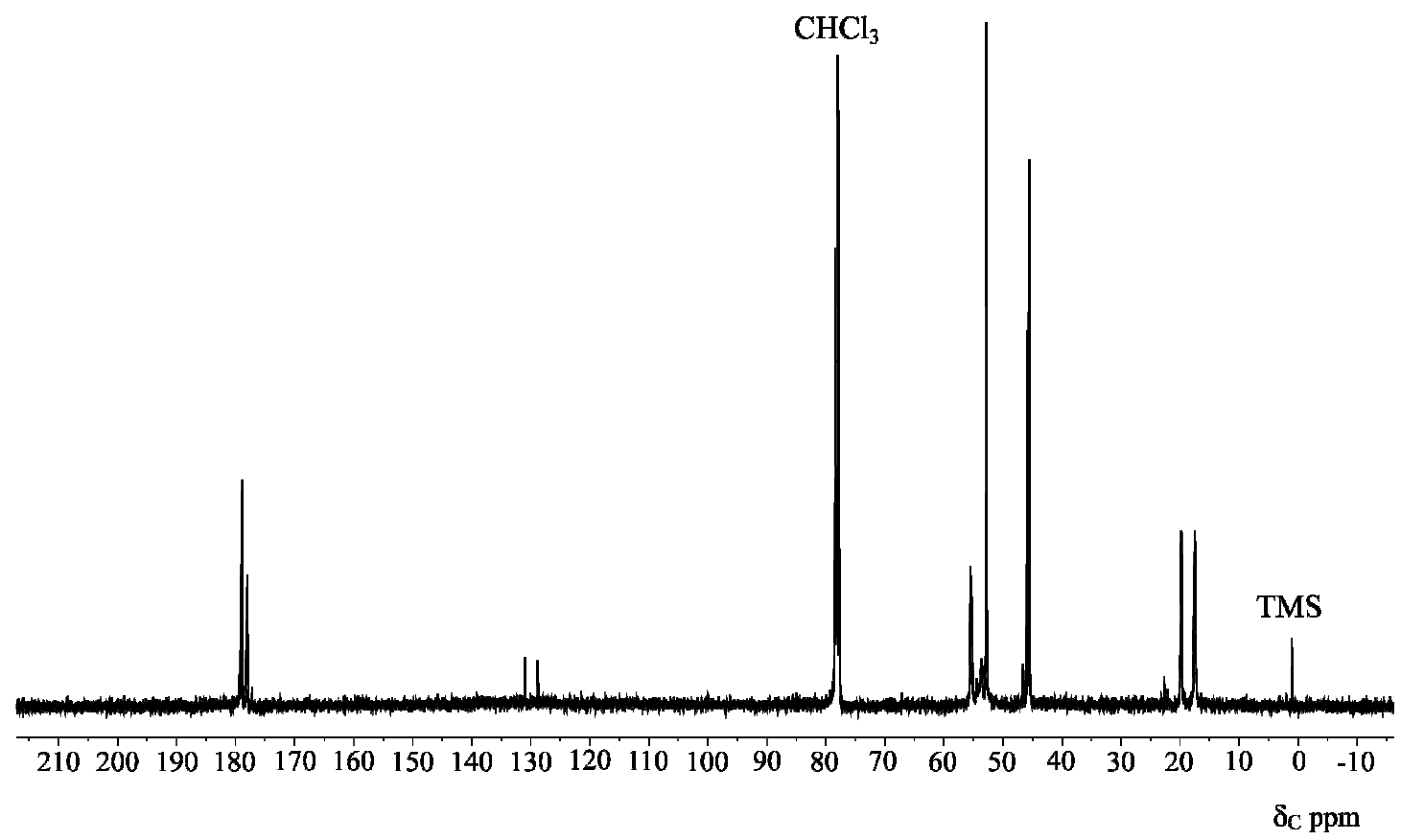

Figure S2. ${ }^{13} \mathrm{C}$ NMR of (PMMA/10 kDa) ${ }_{108}$-PDVB CCS polymer. 